\title{
Analytical design methodology of agent oriented manufacturing systems
}

Patriti V.F., Schäfer K., Charpentier P., Martin P. Université Henri Poincaré, CRAN (Centre de Recherche en Automatique de Nancy) BP 23954506 Vandoeuvre Cedex, France. Phone : (33) 383912000 . Fax: (33) 383912390 \{patriti, schafer, charpentier, martin,\}@cran.u-nancy.fr

\begin{abstract}
Multi-agent manufacturing lacks of design methodology. The aim of this paper is to present a formalisation method that enables the designer to characterise multiagent manufacturing systems in reliability and robustness. This characterisation allows the designer to estimate some characteristics of the system to be designed before its realisation. Finally we present an example of design on the multi-agent machine-tool Shiva.
\end{abstract}

\section{Keywords}

multi-agent, holonic, machine-tool, design, reliability

\section{INTRODUCTION}

The inherent limitations of the original centralised control architecture caused the apparition of agent oriented control paradigms. Multi-Agent, Holonic Manufacturing, Heterarchical and Bionic Systems contribute to agent-oriented manufacturing. Among the numerous questions that arise from these new control paradigms are the problems of agent determination and characterisation of the system. 
At the workshop level, agent determination seems rather straightforward. Indeed, most of the work at the workshop level follow a natural decomposition of the workshop. But as soon as the studied level changes, the typology changes. Thus there are agents for tools (Veeramani \& al., 1993), for the basic components of robots (Regnier \& Duhaut, 1995), flexible cell (Sohier \& al., 1997) and machinetool (Patriti, 1998), scheduling agents (McFarlane \& al., 1995) and watchdog agents (Parunak, 1997). But to help the designer in his choices, few design methodologies adapted to the manufacturing problem are presented, with the exception of Parunak (1997) .

This problem could easily be solved if we could characterised the performances of the overall system. But most of the agent-oriented manufacturing systems properties cannot be evaluated without simulation based on realistic numbers of agents and interchanges (Parunak, 1997). That usually means that the multi-agent control system is already realised. Thus to characterise the system at an early stage of the design process seems to be of great interest and utility. The aim of this paper is to present an analytical modelling technique enabling a designer to characterise his multi-agent control system, thus providing him with information at the specification level. The properties chosen are : reliability and robustness.

\section{THE MULTI-AGENT SYSTEM}

Although our approach could be adapted to other types of multi-agent systems, the analytical model proposed fits a specific type. This chapter presents this type through two different aspects : the relationship between the agents and the physical system on the one hand and their organisation on the other hand.

\section{The agents and the physical system}

A manufacturing system is composed of both a control and an physical system. The physical system transforms, transports and stocks the parts, receives orders from the control system and sends back information. We will name the elements of the physical system components. With an agent oriented approach, the basic element of the control system is called agent. We suppose that :

- Each agent controls one or more components, but the control of one component cannot be shared among several agents.

- Each component or agent is defined by a state variable which equals 1 if the element is functional and 0 if the element is not functional.

Although we do not consider a degraded behaviour within an agent, the degraded behaviour of the system is possible with the re-organisation of the agents.

\section{Organisation}

There are a lot of different methods to make the organisation emerge. Like our model, most of the currently applied methods in manufacturing are inspired by social science. The only assumption this model makes is that the organisation is 
obtained through a service-based protocol. A service is a specification of tasks that the agents are able to provide to other agents.

\section{THE ANALYTICAL MODEL}

The system is composed of :

- A, set of the basic agents.

- $\mathrm{C}$, set of the physical components.

- $F$, set of the services that the components provide. $F=\left\{F_{1}, \ldots, F_{f}\right\}$. $f$ is the number of different services in the system or the cardinal of $\mathrm{F}$.

- $\Omega$, that represents the relationships between agents and components

- $\Phi$, that represents the physical architecture of the system. That is the set of the relationships between the components.

- $\Psi$, that represents the needs of each components. That is the set of the relationships between the services that the components provide and the services they need to realise it.

From these elements, we can compute :

- $\mathbf{S}$, the availability of the services for the components or the agents. That means that $S$ indicates if a service can be used by others or not.

Let MS be the manufacturing system. MS is the six-tuple :

$$
\mathbf{M S}=\langle\mathbf{C}, \mathbf{F}, \mathbf{A}, \Psi, \Omega, \Phi\rangle
$$

The set of the physical components is $C$

$\mathrm{C}=\left\{\mathrm{C}_{1}, \ldots, \mathrm{C}_{\mathrm{c}}\right\} . \mathrm{c}$ is the number of the components in the system or the cardinal of C. $\mathrm{C}$ is composed of the active components like axis, robots and of passive components like tools and parts. The state of component $\mathrm{C}_{\mathrm{i}}$ is defined by $\mathrm{c}_{\mathrm{i}}$.

If $c_{\mathrm{i}}=0$, the component is out of order, it cannot provide any service.

If $c_{\mathrm{i}}=1$, the component is working, it can provide its services if its needs are satisfied.

The set of the basic agents is $A$

$A=\left\{A_{1}, \ldots, A_{a}\right\} . a$ is the number of basic agent in the system or the cardinal of $A$. The state of agent $A_{i}$ is defined by the variable $a_{i}$.

The relationships between agents and components is the matrix $\Omega$

$\Omega$ is a matrix of dimension (a, c) such as :

$\Omega_{\mathrm{i}, \mathrm{j}}=1$, if the component $\mathrm{C}_{\mathrm{j}}$ belongs to agent $\mathrm{A}_{\mathrm{i}}$.

$\Omega_{\mathrm{i}, \mathrm{j}}=0$, if the component $\mathrm{C}_{\mathrm{j}}$ does not belong to agent $\mathrm{A}_{\mathrm{i}}$.

A component can belong to only one agent. 
The state variable of the agents $a_{i}$

Now we can define the state variables of the agents.

If $a_{i}=0$, then the agent $A_{i}$ is down and cannot provide any service ;

If $a_{i}=1$, then the agent is functional and it can provide services if its need are satisfied.

The agent being an aggregation of components, $\mathrm{a}_{\mathrm{i}}$ is a function of $\mathrm{c}_{\mathrm{i}}$ :

$\mathbf{a}_{\mathbf{i}}=\prod \mathbf{c}_{\mathbf{k}}$ such as $\mathrm{C}_{\mathrm{k}}$ is a component of $\mathrm{A}_{\mathrm{i}} \Rightarrow \mathbf{a}_{\mathbf{i}}=\prod_{\mathbf{k}=1}^{\mathbf{k}=\mathbf{c}}\left(\mathbf{c}_{\mathbf{k}}+\overline{\Omega_{\mathbf{i}, \mathbf{k}}}\right)$

Figure 1 presents an example of the relationship between $\mathrm{C}$ and $\mathrm{A}$. There are four components : $\mathrm{C}_{1}, \mathrm{C}_{2}, \mathrm{C}_{3}, \mathrm{C}_{4}$ and two agents $\mathrm{A}_{1}, \mathrm{~A}_{2} . \mathrm{C}_{1}$ and $\mathrm{C}_{2}$ belong to $\mathrm{A}_{1}$, while $\mathrm{C}_{3}$ and $\mathrm{C}_{4}$, belong to $\mathrm{A}_{2}$. That means that :

$\Omega=\left(\begin{array}{llll}1 & 1 & 0 & 0 \\ 0 & 0 & 1 & 1\end{array}\right)$

The architecture is represented by the matrix $\Phi$

While all the components of the system can provide services, there are physical limitations to the co-operation between agents. One of them is the physical architecture of the system. For example a non-mobile robot cannot work with every machine-tool of the workshop but with only those in range. These limitations are modelled with $\Phi . \Phi$ is a matrix of dimension (c, c), c being the number of components in the system.

If $\Phi_{i, j}=1$, the component $C_{j}$ can provide its services to the component $C_{i}$. If $\Phi_{i, j}=0$, the component $C_{j}$ cannot provide its services to the $C_{i}$.

An example of architecture is given in Figure 2. The resulting matrix is :

$$
\Phi=\left(\begin{array}{llll}
0 & 0 & 1 & 1 \\
1 & 0 & 0 & 1 \\
0 & 0 & 0 & 0 \\
0 & 0 & 1 & 0
\end{array}\right) .
$$

The availability of a service for a component is expressed by the matrix $S$ If $S_{i, j}=1$, then the component $\mathrm{C}_{\mathrm{i}}$ may use the service $\mathrm{F}_{\mathrm{j}}$; If $S_{i, j}=0$, then the component $\mathrm{C}_{\mathrm{i}}$ cannot use the service $\mathrm{F}_{\mathrm{j}}$. We will see how to compute the availability later. 


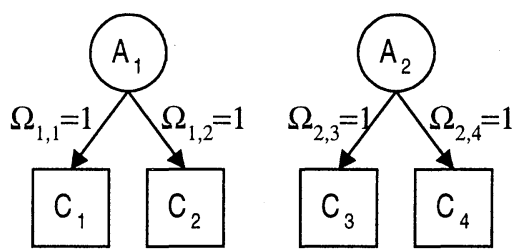

Figure 1 : Example of relationships between $\mathrm{C}$ and $\mathrm{A}$.

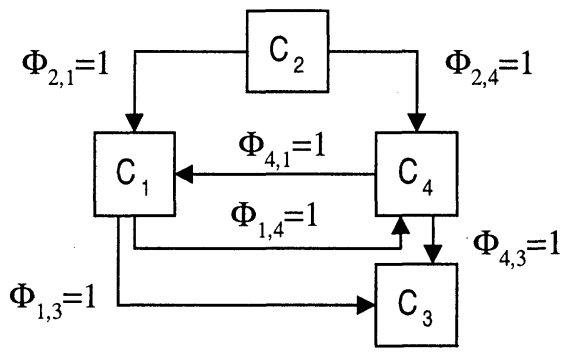

Figure 2 : Example of an architecture.

The need of the physical components are represented by the functions $\Psi_{i, j}$ $\Psi_{i, j}$ are boolean functions of $S_{i}$

If $\psi_{i, j}\left(S_{i}\right)=\psi_{i, j}\left(S_{i, j}, \ldots, S_{i, j}\right)=1$, then the needs of the component $\mathrm{C}_{\mathrm{i}}$ for the service $\mathrm{F}_{\mathrm{j}}$ are fulfilled. Therefore if the component and the associated agent are functional, the service $\mathrm{F}_{\mathrm{j}}$ can be provided.

If $\psi_{\mathrm{ij}}\left(\boldsymbol{S}_{i}\right)=\psi_{\mathrm{ij}}\left(\boldsymbol{S}_{i, t}, \ldots, \boldsymbol{S}_{i, j}\right)=0$, then the needs of the component $\mathrm{C}_{\mathrm{i}}$ for the service $\mathrm{F}_{\mathrm{j}}$ are not fulfilled. Therefore even if the component and the associated agent are functional, the service $F_{j}$ cannot be provided.

For example a tool provides the service machining feature $\mathbf{X}$. Its needs for that service are rotation in $\mathrm{z}$ and translation in $\mathrm{z}$. The boolean function $\Psi$ for the component tool will be :

$\Psi_{\text {tool, machining feature } x}=\mathrm{S}_{\text {rotation in } z} \cdot S_{\text {translation in } z}{ }^{*}$

\section{Availability}

Now we have enough information to compute the availability of the services, which can be done with or without the influence of the control system. In the latter case we will refer to it by the name of potential availability $\mathbf{S p}$ :

$S p_{i, j}=\sum_{\mathbf{k}=1}^{\mathbf{k}=\mathbf{c}} \Phi_{\mathbf{i}, \mathbf{k}} \cdot \mathbf{c}_{\mathbf{k}} \cdot \Psi_{\mathbf{k}, \mathbf{j}}\left(S \mathbf{p}_{\mathbf{k}}\right)$

$S p_{i, j}=1$ means that a service $F_{j}$ is available if the asking component $C_{i}$ is in relation with a provider component $C_{k}$ that is functional, has the service and has its needs satisfied. For the overall system, we have to integrate the effect of the aggregation of components in agents. The availability is then called the overall availability $\mathrm{Sr}$ :

$S r_{i, j}=\sum_{\mathbf{k}=1}^{\mathbf{k}=\mathbf{c} l=\mathbf{l}=\mathbf{a}} \Phi_{\mathbf{l}, \mathbf{k}} \cdot \Omega_{\mathbf{l}, \mathbf{k}} \cdot \mathbf{a}_{\mathbf{l}} \cdot \psi_{\mathbf{k}, \mathbf{j}}\left(\mathbf{S r}_{\mathbf{k}}\right)$. 
$S r_{i, j}=1$ means that the service $F_{j}$ is available to the component $C_{i}$ if the provider agent $A_{1}$ is functional, has a component $C_{k}$ that can realise the service, has its needs satisfied and is in relation with $C_{i}$.

\section{CHARACTERISING THE SYSTEM}

The former model enables us to build the overall properties of the system from the distributed models of each component and agent. This is based on the theory of reliability of non-renewable components (Bon, 1995). The properties can be computed for both the overall system and the potential system. The overall system integrates the influences of the control system (the agents), while the potential system does not.

\section{Reliability}

The designer may compute the reliability of any service in the system. In a manufacturing system the most important service is associated with the product : manufacture the product. The reliability of the potential system is defined as the probability that the service would be realised without the influence of the control system. The formal definition is then :

$$
\mathbf{R}_{\mathbf{p}}=\mathbf{P}\left[\Psi_{\text {manufacture_product }}\left(\mathbf{S p}_{\mathbf{i}}\right) \cdot \mathbf{c}_{\mathbf{i}}=1\right] .
$$

If there are more than one product in the system, or if there is an inexhaustible amount of raw material at disposal, we can assume that $c_{i}=1$. The reliability of the overall system integrates the influence of the agents in the probability. Its formal definition is then :

$$
\mathbf{R}_{\mathbf{r}}=\mathbf{P}\left[\Psi_{\text {manufacture_product }}\left(\mathbf{S r}_{\mathbf{i}}\right) \cdot\left(\sum_{\mathbf{j}=1}^{\mathbf{a}} \Omega_{\mathbf{j}, \mathbf{i}} \cdot \mathbf{a}_{\mathbf{j}}\right)=1\right] .
$$

\section{Robustness}

The designer may also compute the robustness of any service in the system. We give our own definition of this property based on the notion of the critical path :

The path $\mathrm{Pa}_{\mathrm{S}_{\mathrm{i}}}$ for $\phi\left(\mathrm{S}_{\mathrm{i}}\right)=1$ is a set, such as: $\mathbf{P a}_{\mathbf{S i}}=\left\{\mathrm{j}: \mathrm{S}_{\mathrm{i}, \mathrm{j}}=1\right\}$.

$\mathrm{Pa}_{\mathrm{S}_{\mathrm{i}}}$ is a critical path if $: \forall \mathbf{S}_{\mathbf{k}}<\mathbf{S}_{\mathbf{i}} \Rightarrow \phi\left(\mathbf{S}_{\mathbf{k}}\right)=0$.

The robustness of a system $\mathrm{H}$ is defined as the number or critical paths of realisation of the service. Its formal definition is then :

$$
\mathbf{H}=\operatorname{card}\left\{\mathbf{P a}_{\mathbf{S}_{\mathbf{i}}}: \phi\left(\mathbf{S}_{\mathbf{i}}\right)=1, \forall \mathbf{S}_{\mathbf{k}}<\mathbf{S}_{\mathbf{i}} \Rightarrow \phi\left(\mathbf{S}_{\mathbf{k}}\right)=0\right\}
$$


Because this is a manufacturing system, the service used is again manufacture the product. That means that the robustness of the potential system $\mathrm{Hp}$ is computed for :

$$
\phi\left(\mathbf{S}_{\mathbf{i}}\right)=\Psi_{\text {manufacture_product }}\left(\mathbf{S}_{\mathbf{i}}\right) \cdot \mathbf{c}_{\mathbf{i}}=1 .
$$

And that the robustness of the overall system is computed for :

For $\phi\left(\mathbf{S}_{\mathbf{i}}\right)=\Psi_{\text {manufacture_product }}\left(\mathbf{S}_{\mathbf{i}}\right) \cdot\left(\sum_{\mathbf{j}=1}^{\mathbf{a}} \Omega_{\mathbf{j}, \mathbf{i}} \cdot \mathbf{a}_{\mathbf{j}}\right)=1$.

\section{DESIGNING A SYSTEM}

Two policies are then available for a designer. First use the analytical formalism to search the solution space and generate the optimal solutions with an optimisation algorithm. Second generate manually several solutions and compare the emergent properties to choose the best one. These steps do not exclude simulation, in fact one of the most important properties of a manufacturing system, namely productivity, is not covered by this formalism and still needs a validation through simulation.

The following design is based on Shiva (Figure 3) a multi-agent machine-tool built at the CRAN (Centre of Research for Automatic Control in Nancy). Shiva is basically a double milling machine with two autonomous three axis workstations.

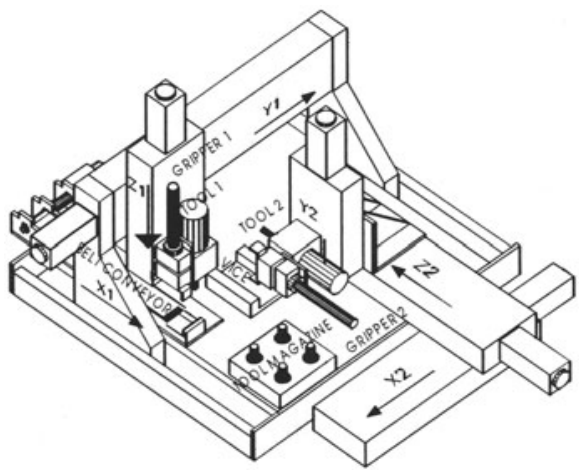

Figure 3 : Shiva.

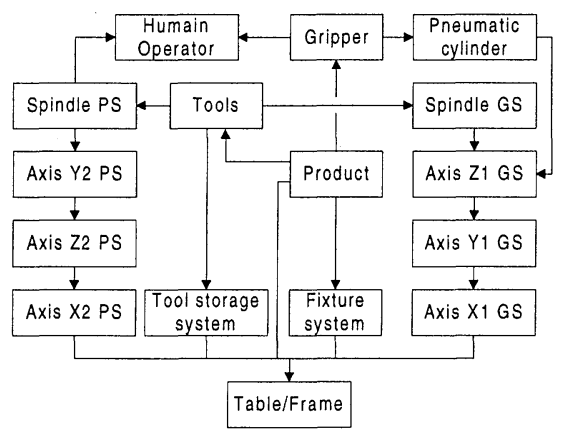

Figure 4 : Architecture of Shiva.

\subsection{The physical system}

The architecture of Shiva is given in Figure 4. As we can see there are six linear axis grouped in two stations (PS and GS). The workstation GS has in addition to the spindle a gripper with a roto-linear pneumatic cylinder that enables it to 
transport workpieces. If the gripper is occupied otherwise, the human operator can move the product himself. Because of its vertical position the GS spindle is able to change its tool without manual aid, while the PS spindle must use the human operator.

The set $\mathrm{C}$ is composed of 21 elements :

$\mathrm{C}=\{$ Table, Tool Storage System, Fixture System, Spindle GS, Spindle PS, Gripper, Cylinder, Axis 1 GS, Axis 2 GS, Axis 3 GS, Axis 1 PS, Axis 2 PS, Axis 3 PS, Product, Tool1, Tool2, Tool3, Tool4, Tool5, Tool6, Operator .

The set $\mathrm{F}$ is composed of 26 services :

$\mathrm{F}=\{\operatorname{roughing}(-\mathrm{x})$, roughing $(\mathrm{z})$, semi-finishing $(-\mathrm{x})$, semi-finishing $(\mathrm{z})$, finishing($\mathrm{x})$, finishing(z), stock_tool, machining(-x), machining(z), move_tool, move(x), move(y), move(z), stock_component, Fixture A, Fixture B, Fixture C, Fixture D, Fixture E, stock_product, rotation $(\mathrm{x})$, rotation(y), rotation(z), transport_product, translation(z), manufacture_product $\}$.

Roughing, semi-finishing and finishing are machining operations. They are given for a specific direction. All these services are provided by the tools. Machining is the service provided by the spindles for the tools. This service combines rotation and movement. Rotation, translation and transport_product are transport services provided by the cylinder and the gripper for the product. Stock_tool and move_tool are services provided for the tools to move them in and out of the tool storage system. Fixtures are the services given by the fixturing system. Each different fixture represents a different position of the part. Moves are the services provided by the axis. They represent controlled linear movements. Stock_components is the service provided by every component that physically supports another component. For example the axis Y1 GS is supported by the axis X1 GS. Stock_product is the service provided by the table. To give all the $\Psi$ functions would exceeds the authorised length of this paper, so we limit the description to the most important functions.

\section{Product}

The main function $\Psi$ is the function of the product: manufacture_product. This function gives the structure of the chosen process plan.

$\Psi=$ Stock_Product. Transport_Product. \{Fixture A. Roughing(-x). SemiFinishing($x)$. Finishing(-x). Roughing(z). SemiFinishing(z). Finishing(z) + Fixture B: Fixture C . Roughing(z). SemiFinishing $(z)$. Finishing $(z)+$ Fixture D. Fixture E. Roughing(-x). SemiFinishing(-x). Finishing(-x) \}. 
Tool

To realise its function, the component part needs the services of the tools. The function $\Psi$ of the service Roughing(-x) for the tool is the following :

$\Psi=$ Machining(-x). Stock_tool.

\subsection{The potential system}

Using each function of each component and comparing them with the architecture $\Phi$, we can reconstruct the success conditions of manufacture_the_product according to the state variables of the components. The corresponding reliability block diagram (Bon, 1995) is represented Figure 5.

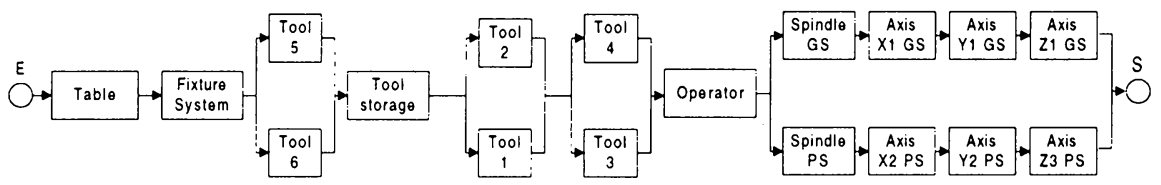

Figure 5 : Reliability block diagram of the potential system.

Then we can deduce the potential reliability $\mathrm{Rp}$ and the potential robustness $\mathrm{Hp}$ :

$\mathbf{H}_{\mathrm{p}}=16$

$\mathbf{R}_{\mathbf{p}}=\mathbf{R}_{\text {Fixture }} \cdot \mathbf{R}_{\text {Tool storage }} \cdot \mathbf{R}_{\text {Operator }} \cdot \mathbf{R}_{\text {Table }} \cdot\left(\mathbf{R}_{\text {Tool5 }}+\mathbf{R}_{\text {Tool6 }}-\mathbf{R}_{\text {Tool5 } 5} \cdot \mathbf{R}_{\text {Tool6 }}\right)$.

$\left(\mathbf{R}_{\text {Tool1 }}+\mathbf{R}_{\text {Tool2 }}-\mathbf{R}_{\text {Tool1 } 1} \cdot \mathbf{R}_{\text {Tool2 }}\right) \cdot\left(\mathbf{R}_{\text {Tool4 }}+\mathbf{R}_{\text {Tool5 }}-\mathbf{R}_{\text {Tool4 }} \cdot \mathbf{R}_{\text {Tool5 }}\right)$.

$\left(\mathbf{R}_{\text {SpindleGS }} \cdot \mathbf{R}_{\text {AxisX1GS }} \cdot \mathbf{R}_{\text {AxisY1GS }} \cdot \mathbf{R}_{\text {AxisZ1GS }}\right.$

$+\mathbf{R}_{\text {SpindlePS }} \cdot \mathbf{R}_{\text {AxisX2PS }} \cdot \mathbf{R}_{\text {Axis } 22 P S} \cdot \mathbf{R}_{\text {AxisZ2PS }}$

$\left.-\mathbf{R}_{\text {SpindleGS }} \cdot \mathbf{R}_{\text {AxisXIGS }} \cdot \mathbf{R}_{\text {AxisY1GS }} \cdot \mathbf{R}_{\text {AxisZlGS }} \cdot \mathbf{R}_{\text {SpindlePS }} \cdot \mathbf{R}_{\text {AxisX2PS }} \cdot \mathbf{R}_{\text {AxisY2PS }} \cdot \mathbf{R}_{\text {AxisZ2PS }}\right)$

\section{Finding the agents}

Finding the agents can be seen as the determination of $\Omega$ that maximises $\mathrm{Hr}$ and $\mathrm{Rr}$. We have decided not to use an optimisation algorithm, but to make a heuristic search by comparing possible structures. Figure 6 shows the result: Three basic agents are composed of more than one component. The agent GS is composed of the axis X1, Y1 and $\mathbf{Z 1}$. The agent PS is composed of the axis $\mathbf{X 2 ,} \mathbf{Y 2}$ and $\mathbf{Z 2}$. The agent Transport is composed of the Gripper and the Pneumatic cylinder. The other basic agents consist of only one component. Thus $\mathrm{Hr}$ and $\mathrm{Rr}$ are the same than $\mathrm{Hp}$ and Rp. Shiva was then simulated to evaluate the productivity of the control architecture. This specification was programmed using the contract-net protocol for the control system of the machine-tool Shiva.

\section{CONCLUSION}

We have presented a design methodology based on a formalism, which enables the designer to evaluate the reliability and the robustness of a system at the 
specification level. Finally we have shown an application of this methodology through the design of the agents of the machine-tool Shiva.

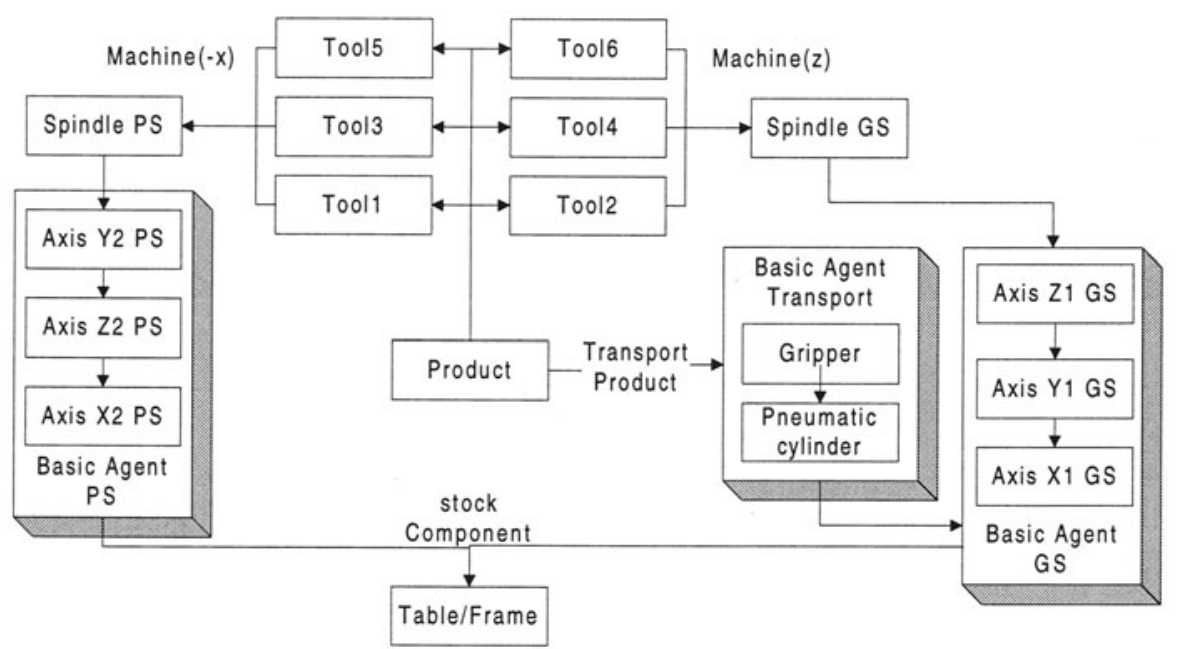

Figure 6 : The chosen architecture.

\section{REFERENCES}

Bon J.L., (1995) Fiabilité des systèmes, méthodes mathématiques, Masson, Paris.

MacFarlane D., Marett B., Elsley G., Jarvis D. (1995) Application of holonic methodologies to problem diagnosis in a steel rod mill. IEEE Conference on systems man and cybernetics, october 95, Vancouver, 940-945.

Patriti V. (1998) Systèmes de pilotage auto-organisés et gammes distribuées: méthode de conception et application à une machine-outil, University Henri Poincaré-Nancy I thesis, 16/01/98.

Parunak H.V.D., Sauter J., Clark S. (1997) Toward the specification and design of industrial synthetic ecosystems. Workshop on Agent theories, architectures and languages: ATAL'97.

Regnier S., Duhaut D. (1995), Une approche multi-agents pour la résolution de problèmes robotiques. Troisième journées francophones sur l'IAD et les SMA, Saint bandolf, savoie, France, 15-17 March, 91-97.

Sohier C. , Denis B., Vigny C. (1997) Validation du comportement dynamique d'un système multi-agents : application de l'éco-résolution à une cellule de production manufacturière. Actes du Congrès MOSIM'97 : Systèmes de Production et de Logistique, Rouen, France, 5-6 juin 1997.

Veeramani D., Bhargava B., Barash M.M. (1993) Information system architecture for heterarchical control of large FMSs. Computer Integrated Manufacturing Systems, Vol. 6, No 2, Mai, 1993. 